
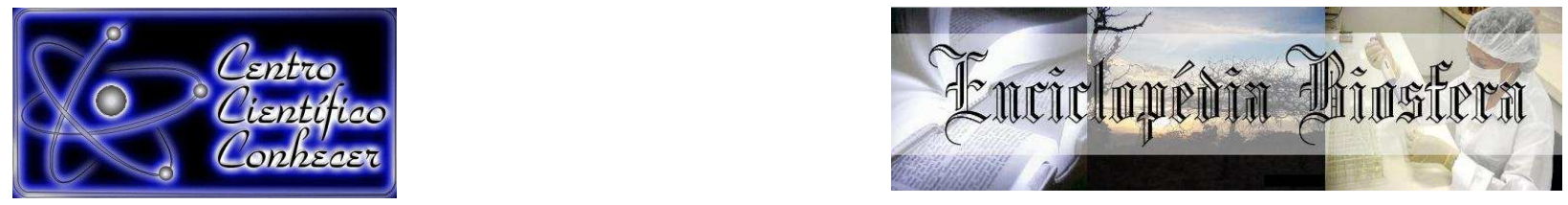

\title{
AVALIAÇÃO DO CRESCIMENTO E DESENVOLVIMENTO DE Toona ciliata VAR. AUSTRALIS, EM DIFERENTES SUBSTRATOS E RECIPIENTES
}

Lúcia Vânia Ribeiro Lisboa', Patrícia Aparecida de Souza², Douglas Santos Gonçalves $^{3}$, Priscila Bezerra da Silva², Katiane Sousa Carvalho ${ }^{1}$

1 Graduanda em Engenharia Florestal da Universidade Federal do Tocantins (lucia_vania010@hotmail.com), Gurupi - Brasil

2 Professora Dra. do departamento de Engenharia Florestal da Universidade Federal do Tocantins, Gurupi - Brasil

3 Mestrando em Ciências Florestais e Ambientais da Universidade Federal do Tocantins, Gurupi - Brasil

Recebido em: 08/04/2016 - Aprovado em: 30/05/2016 - Publicado em: 20/06/2016 DOI: 10.18677/Enciclopedia_Biosfera_2016_015

\begin{abstract}
RESUMO
Entre as espécies exóticas que vem sendo plantadas no Brasil, destaca-se a Toona ciliata var. australis, pertence à família Meliaceae, e que possui características interessantes para o setor madeireiro. O presente trabalho teve por objetivo avaliar o desenvolvimento das mudas em relação aos substratos e recipientes e avaliar as características morfológicas mudas de Toona ciliata var. australis. As mudas foram produzidas em dois grupos de recipientes com tubetes $\left(50,110\right.$ e $\left.175 \mathrm{~cm}^{3}\right)$ e sacos plásticos $(17 \times 22,18 \times 30$ e $25 \times 30 \mathrm{~cm})$, onde os substratos capim-mombaça $(\mathrm{CM})$, esterco bovino (EB) e esterco de galinha (EG), foram associados em iguais proporções formando o composto orgânico; Terra de subsolo (TS) e substrato comercial Bioflora (SC), constituindo-se dois experimentos instalados concomitantemente. $\mathrm{O}$ tratamento que proporcionou os maiores resultados para todas as características analisadas foi o T17, que continha como substrato o composto orgânico (capim-mombaça + esterco de galinha + esterco bovino) e o recipiente saco plástico (18×30).
\end{abstract}

PALAVRAS-CHAVE: Cedro Australiano, produção de mudas, viveiro florestal.

\section{EVALUATION OF GROWTH AND DEVELOPMENT OF Toona ciliata VAR. AUSTRALIS IN DIFFERENT SUBSTRATES AND RECIPIENTS}

\begin{abstract}
Among the exotic species that have been planted in Brazil, there is the Toona ciliata var. australis belongs to the Meliaceae family, and has interesting features for the timber sector. This study aimed to evaluate the development of seedlings in relation to substrates and containers and evaluate the morphological characteristics in Toona ciliata var. australis. The seedlings were grown in two groups of containers with tubes $\left(50,110\right.$ and $\left.175 \mathrm{~cm}^{3}\right)$ and plastic bags $(17 \times 22,18 \times 30$ and $25 \times 30 \mathrm{~cm})$, where the
\end{abstract}


substrates mombaça (CM), manure (EB) and chicken manure (EG), they have been associated in equal proportions forming the organic compound; subsoil (TS) and commercial substrate Bioflora (SC), constituting two concurrently installed experiments. The treatment gave the highest results for all characteristics was the T17, which contained substrate as the organic compound (mombaça + chicken manure + cattle manure) and the container plastic bag $(18 \times 30)$. KEYWORDS: Cedar Australian, Containers, substrates.

\section{INTRODUÇÃO}

No Brasil, os estudos silviculturais têm se voltado, quase que exclusivamente, para as espécies de rápido crescimento, como, por exemplo, as do gênero Eucalyptus e Pinus. Entretanto, outras espécies devem ser estudadas visando a uma maior diversificação. Uma espécie que tem se mostrado bastante promissora, é a Toona ciliata var. australis conhecida popularmente como cedro-australiano (MORETTI et al., 2011).

A Toona ciliata var. australis é uma espécie nativa da Austrália tropical, pertencente à família botânica Meliaceae (MIGLIORINI et al., 2015). Possui características interessantes para o setor madeireiro, apresenta rápido crescimento e produz madeira de boa qualidade, com características tecnológicas semelhantes ao cedro brasileiro (Cedrela odorata) (BRAZ et al., 2013). Sua madeira é rica em extrativos, os quais podem auxiliar no aumento da durabilidade natural, possui elevada resistência ao broqueador das pontas (Hypsipyla grandella Zeller), que afeta os cedros nativos (ALMEIDA et al., 2012). Por esses motivos é de grande importância conhecer todas as características de desenvolvimento da espécie, iniciando pelo processo de produção de mudas.

Os primeiros aspectos que devem ser avaliados para a produção de mudas de boa qualidade são os tipos de substratos e o tamanho do recipiente (COÊLHO et al., 2013). Pois, estes apresentam influência direta na qualidade e no custo final das mudas produzidas (BARBOSA et al., 2013). O tamanho do recipiente deve permitir o desenvolvimento da raiz sem restrições durante o período de permanência no viveiro, deve conter substrato que permita o crescimento e nutrição das mudas; promover adequada formação do sistema radicular e proteger as raízes de danos mecânicos e da desidratação; e contribuir para a máxima sobrevivência e crescimento inicial no campo. Os recipientes devem apresentar dimensões uniformes; ser facilmente manuseáveis no viveiro, no transporte e no plantio; possibilitar a mecanização das operações de enchimento, semeadura no viveiro e plantio no campo (LISBOA et al., 2012).

Entre os recipientes mais utilizados para produção de mudas nativas estão os tubetes e os sacos plásticos (ABREU et al., 2015). Embora, haja uma forte disposição ao uso de tubetes rígidos como recipiente, o uso de sacos de polietileno, ainda é muito empregado na produção de mudas de espécies florestais (ALVES et al., 2012). Estas apresentam maior disponibilidade no mercado, menor custo de aquisição e baixo investimento em infra-estrutura de viveiros, sendo utilizados pelos viveiristas que produzem pequenas quantidades de mudas (AJALA, 2012).

Um dos fatores de grande influência na produção de mudas está relacionado com a escolha adequada do substrato que será utilizado (OLIVEIRA et al., 2014). O estudo do substrato é o aspecto de fundamental importância porque ele exerce uma 
influência marcante na arquitetura do sistema radicular e no estado nutricional das plantas, afetando profundamente a qualidade das mudas (COÊLHO et al., 2013).

A utilização de substratos alternativos para a produção de mudas podem ser formados pela combinação de diversos componentes ou por um único material, podendo ser de origem vegetal, mineral ou sintética (BOENE et al., 2013). Estes vêm sendo aplicados, visando oferecer alternativas para produtores de mudas (KLEIN, 2015).

O fornecimento de novos produtos a serem utilizados como substratos, apresentam novas alternativas de formulação (KRATZ, et al., 2013), com o objetivo de aprofundar conhecimentos sobre alternativas viáveis para a produção de substratos em substituição aos substratos comerciais (KLEIN, 2015). A terra de subsolo pode ser considerada a opção mais requisitada para formação de mudas, por estar isenta de sementes de plantas indesejáveis e de microrganismos patogênicos. Porém podem apresentar baixa fertilidade ou desequilíbrio nutricional (SILVA \& MORAIS, 2013).

Diante dessas considerações e do potencial da Toona ciliata var. australis para plantios comerciais que visem à produção de madeira, ressalta-se que é de grande importância o conhecimentos em relação ao crescimento e desenvolvimento da espécie. Portanto, o presente trabalho teve por objetivo avaliar o desenvolvimento das mudas da espécie em diferentes substratos e recipientes.

\section{MATERIAL E MÉTODOS}

Para a realização do presente trabalho desenvolveram-se dois experimentos no Viveiro Florestal da Universidade Federal do Tocantins (UFT), Gurupi - TO, apresentando coordenadas geográficas de 1143'45" S e 4904'07" W, com altitude média de $280 \mathrm{~m}$.

As mudas de Toona ciliata foram produzidas em dois grupos de recipientes com tubetes e sacos plásticos, constituindo-se dois experimentos instalados concomitantemente (tabela 1). Para cada experimento, utilizou-se o delineamento em DIC (delineamento inteiramente causalisado), fatorial $(3 \times 6), 3$ substratos e 6 recipientes, constituindo-se 18 tratamentos, com 6 repetições e 5 sementes por repetição. As médias dos tratamentos foram realizadas através do Software ASSISTAT versão 7.7 beta (SILVA, 2015), pelo teste TUKEY (1977) a nível de 5\% de probabilidade.

Nos dois experimentos, foram utilizados nos tratamentos os seguintes substratos: capim-mombaça $(\mathrm{CM})$ + esterco bovino $(\mathrm{EB})$ + esterco de galinha (EG), estes foram associados em iguais proporções formando o composto orgânico $(\mathrm{CM}+\mathrm{EB}+\mathrm{EG})$; Terra de subsolo (TS) e substrato comercial Bioflora (SC), (Tabela 1). 
TABELA 1: Tratamentos utilizados de acordo com os recipientes e substratos avaliados.

\begin{tabular}{|c|c|c|c|}
\hline Tratamentos & Recipientes & Substratos & № Repetições \\
\hline $\mathrm{T} 1$ & & Subsolo & 6 \\
\hline T2 & Tubete $\left(50 \mathrm{~cm}^{3}\right)$ & Orgânico & 6 \\
\hline T3 & & Comercial & 6 \\
\hline T4 & & Subsolo & 6 \\
\hline T5 & Tubete $\left(110 \mathrm{~cm}^{3}\right)$ & Orgânico & 6 \\
\hline T6 & & Comercial & 6 \\
\hline T7 & & Subsolo & 6 \\
\hline T8 & Tubete $\left(175 \mathrm{~cm}^{3}\right)$ & Orgânico & 6 \\
\hline T9 & & Comercial & 6 \\
\hline$T 10$ & & Subsolo & 6 \\
\hline T11 & Saco Plástico (17x22) & Orgânico & 6 \\
\hline T12 & $4991,0 \mathrm{~cm}^{3}$ & Comercial & 6 \\
\hline T13 & & Subsolo & 6 \\
\hline T14 & Saco Plástico (18×30) & Orgânico & 6 \\
\hline T15 & $7630,2 \mathrm{~cm}^{3}$ & Comercial & 6 \\
\hline T16 & & Subsolo & 6 \\
\hline T17 & Saco Plástico (25×30) & Orgânico & 6 \\
\hline T18 & $14718,75 \mathrm{~cm}^{3}$ & Comercial & 6 \\
\hline
\end{tabular}

As sementes de Toona ciliata var. australis foram adquiridas através da Empresa Sementes Caiçara. O experimento foi instalado no dia 25 de junho de 2015. As mudas foram acondicionadas, no viveiro florestal (com sombrite de $50 \%$ ), sendo irrigadas duas vezes ao dia, por sistema de irrigação manual. Após 60 dias foi realizado o raleio dessas, deixando sempre a maior e mais centralizada.

Devido a um lento desenvolvimento das mudas em relação aos tratamentos utilizados, as avalições de diâmetro do colo (com auxílio de um paquímetro, graduado em $\mathrm{mm}$ ) e altura (com auxílio de uma régua, graduada em centímetros) foram realizadas aos 110, 140 e 170 dias após a semeadura. Visando avaliar somente a influência dos substratos na nutrição das mudas em diferentes recipientes com diferentes volumes, não houve qualquer adubação de base e de cobertura.

Aos 170 dias, no final do experimento foram obtidos os parâmetros, comprimento de raiz, massa seca radicular (MSR) e massa seca da parte aérea (MSPA), onde, as raízes foram separadas da parte aérea, lavadas em peneiras e, em seguida, foram acondicionadas em sacos de papel Kraft e colocadas para secar em estufa com circulação forçada de ar, a $70^{\circ} \mathrm{C}$ até a obtenção de peso constante. O Índice de Qualidade de Dickson (IQD) (DICKSON et al., 1960), calculado através da fórmula a baixo: 


$$
\mathrm{IQD}=\frac{\mathrm{MST}(\mathrm{g})}{\mathrm{H}(\mathrm{cm})} \frac{\mathrm{PMSP}(\mathrm{g})}{\mathrm{DC}(\mathrm{mm})}
$$

As médias dos tratamentos foram realizadas através do Software ASSISTAT versão 7.7 beta (SILVA, 2015), pelo teste Tukey ao nível de $5 \%$ de probabilidade.

\section{RESULTADOS E DISCUSSÃO}

As mudas de Toona ciliata var. australis apresentaram diferenças de crescimento em diâmetro e altura entre os tratamentos testados (tabela 2).

TABELA 2: Médias de altura de plantas $(H)$ e diâmetro de colo (DC) de mudas de Toona ciliata var. australis produzidas em diferentes substratos e recipientes, medidas aos 110, 140, e 170 dias após a semeadura (DAS).

\begin{tabular}{|c|c|c|c|c|c|c|}
\hline \multirow[t]{2}{*}{ TRATAMENTO } & \multicolumn{2}{|c|}{110 DAS } & \multicolumn{2}{|c|}{140 DAS } & \multicolumn{2}{|c|}{170 DAS } \\
\hline & $H(\mathrm{~cm})$ & $\begin{array}{c}\mathrm{DC} \\
(\mathrm{mm})\end{array}$ & $H(\mathrm{~cm})$ & $\mathrm{DC}(\mathrm{mm})$ & $H(\mathrm{~cm})$ & $\mathrm{DC}(\mathrm{mm})$ \\
\hline T1 & $0,00 \mathrm{~b}$ & $0,00 \mathrm{~d}$ & $0,00 \mathrm{c}$ & $0,00 \mathrm{~b}$ & $0,00 \mathrm{c}$ & $0,00 \mathrm{e}$ \\
\hline T2 & $2,54 \mathrm{~b}$ & $0,10 \mathrm{c}$ & $0,00 \mathrm{c}$ & $0,00 \mathrm{~b}$ & $0,00 \mathrm{c}$ & $0,00 \mathrm{e}$ \\
\hline T3 & $1,72 \mathrm{~b}$ & $0,10 \mathrm{c}$ & $2,05 \mathrm{c}$ & $0,10 \mathrm{~b}$ & $2,50 \mathrm{c}$ & $0,10 \mathrm{de}$ \\
\hline T4 & $0,00 \mathrm{~b}$ & $0,00 \mathrm{~d}$ & $0,00 \mathrm{c}$ & $0,00 \mathrm{~b}$ & $0,00 \mathrm{c}$ & $0,00 \mathrm{e}$ \\
\hline T5 & $2,24 \mathrm{~b}$ & $0,10 \mathrm{c}$ & $3,35 c$ & $0,10 \mathrm{~b}$ & $0,00 \mathrm{c}$ & $0,00 \mathrm{e}$ \\
\hline T6 & $1,98 \mathrm{~b}$ & $0,10 \mathrm{c}$ & $2,00 \mathrm{c}$ & $0,10 \mathrm{~b}$ & $2,70 \mathrm{c}$ & $0,10 \mathrm{de}$ \\
\hline T7 & $0,00 \mathrm{~b}$ & $0,00 \mathrm{~d}$ & $0,00 \mathrm{c}$ & $0,00 \mathrm{~b}$ & $0,00 \mathrm{c}$ & $0,00 \mathrm{e}$ \\
\hline T8 & $4,30 \mathrm{~b}$ & $0,20 \mathrm{bc}$ & $5,20 \mathrm{c}$ & $0,20 \mathrm{~b}$ & $6,60 \mathrm{c}$ & $0,20 \mathrm{~cd}$ \\
\hline T9 & $2,00 \mathrm{~b}$ & $0,10 \mathrm{c}$ & $2,20 \mathrm{c}$ & $0,10 \mathrm{~b}$ & $2,38 \mathrm{c}$ & $0,10 \mathrm{de}$ \\
\hline T10 & $1,36 \mathrm{~b}$ & $0,10 \mathrm{c}$ & $1,60 \mathrm{c}$ & $0,14 \mathrm{~b}$ & $1,88 \mathrm{c}$ & $0,14 \mathrm{~d}$ \\
\hline $\mathrm{T} 11$ & $1,03 \mathrm{a}$ & $0,26 \mathrm{ab}$ & $15,61 \mathrm{~b}$ & $0,46 \mathrm{a}$ & $24,21 b$ & $0,63 \mathrm{~b}$ \\
\hline T12 & $1,93 \mathrm{~b}$ & $0,10 \mathrm{c}$ & $2,58 \mathrm{c}$ & $0,10 \mathrm{~b}$ & $4,28 \mathrm{c}$ & $0,14 \mathrm{~d}$ \\
\hline T13 & $1,45 \mathrm{~b}$ & $0,10 \mathrm{c}$ & $1,54 \mathrm{c}$ & $0,10 \mathrm{~b}$ & $2,00 \mathrm{c}$ & $0,10 \mathrm{de}$ \\
\hline T14 & $14,05 \mathrm{a}$ & $0,35 \mathrm{a}$ & $25,23 \mathrm{a}$ & $0,60 \mathrm{a}$ & $34,13 \mathrm{a}$ & $0,83 \mathrm{a}$ \\
\hline T15 & $1,91 \mathrm{~b}$ & $0,10 \mathrm{c}$ & $2,20 \mathrm{c}$ & $0,13 \mathrm{~b}$ & $2,82 \mathrm{c}$ & $0,15 \mathrm{~cd}$ \\
\hline T16 & $1,55 \mathrm{~b}$ & $0,10 \mathrm{c}$ & $1,96 \mathrm{c}$ & $0,11 \mathrm{~b}$ & $2,21 \mathrm{c}$ & $0,11 \mathrm{de}$ \\
\hline T17 & $10,66 \mathrm{a}$ & $0,30 a b$ & $20,40 a b$ & $0,60 \mathrm{a}$ & $35,76 \mathrm{a}$ & $0,93 \mathrm{a}$ \\
\hline T18 & $2,18 b$ & $0,11 \mathrm{c}$ & $3,51 \mathrm{c}$ & $0,11 \mathrm{~b}$ & $7,05 \mathrm{c}$ & $0,26 \mathrm{c}$ \\
\hline Média & 3,47 & 0,12 & 5,42 & 0,17 & 8,09 & 0,23 \\
\hline CV (V\%) & 50,20 & 33,19 & 50,04 & 43,63 & 40,30 & 22,82 \\
\hline
\end{tabular}

As médias seguidas pela mesma letra não diferem estatisticamente entre si pelo teste de Tukey ao nível de $5 \%$ de probabilidade.

$\mathrm{Na}$ análise estatística observaram-se diferenças entre os tipos de substratos e recipientes, sendo significativas para todas as características analisadas. Notou-se que a altura da muda foi influenciada pelos diferentes substratos e recipientes, constatou-se que as plantas provenientes de tratamento T14 (Substrato orgânico e 
recipiente de $18 \times 30 \mathrm{~cm}$ ) e T17 (Substrato orgânico e recipiente de $25 \times 30 \mathrm{~cm}$ ) atingiram, aos 170 dias, altura média superior a $34 \mathrm{~cm}$. Isto, provavelmente se deve a uma maior quantidade de nutrientes disponível para as mudas e espaço adequado para o seu desenvolvimento, ou seja, sacos plásticos de $18 \times 30$ e 25×30 cm, (tabela 2).

Considerando a altura das mudas de Toona ciliata var. australis, observou-se que os valores médios de altura da parte aérea, aos 110 dias após a semeadura nos tratamentos T11(Substrato orgânico e recipiente de $17 \times 22 \mathrm{~cm}$ ), T14 (Substrato orgânico e recipiente de $18 \times 30 \mathrm{~cm}$ ) e T17 (Substrato orgânico e recipiente de 25×30 $\mathrm{cm}$ ), e aos 140 dias o tratamento T14 (Substrato orgânico e recipiente de $18 \times 30 \mathrm{~cm}$ ) e aos 170 dias os tratamentos T14 (Substrato orgânico e recipiente de 18x30 cm) e T17 (Substrato orgânico e recipiente de $25 \times 30 \mathrm{~cm}$ ), não apresentaram diferenças significativas entre si, onde obtiveram maiores médias de crescimento, diferenciando-se estatisticamente dos demais tratamentos (tabela 2).

Em relação ao efeito do tamanho do recipiente sobre a altura das plantas, pôde-se inferir neste trabalho, que o maior desenvolvimento das mudas ocorreu em recipientes de maiores dimensões, nos tratamentos T14 (Substrato orgânico e recipiente de $18 \times 30 \mathrm{~cm}$ ) e T17 (Substrato orgânico e recipiente de $25 \times 30 \mathrm{~cm}$ ). Resultados semelhantes aos encontrados por outros autores, com diferentes espécies arbóreas.

SOUZA et al., (2005) e CUNHA et al., (2005), avaliaram quatro tamanhos de recipientes para produção de mudas de Tabebuia serratifolia e Tabebuia impetiginosa, os mesmos, constataram, que os recipientes de maiores dimensões proporcionaram tendência ao maior crescimento das mudas. O substrato que proporcionou os melhor resultados para altura continha em sua composição, esterco bovino + esterco de galinha + capim-mombaça. De acordo com CUNHA et al., (2005) o efeito significativamente positivo do substrato enriquecido com composto orgânico no crescimento em altura de mudas pode estar relacionado com a maior disponibilidade de $\mathrm{P}, \mathrm{Ca}, \mathrm{Mg}$ e $\mathrm{K}$ e com $\mathrm{o} \mathrm{pH}$, situado em níveis adequados ao desenvolvimento das plantas.

Considerando o diâmetro de colo das mudas cultivadas aos 110 dias após a semeadura no tratamento T14 (Substrato orgânico e recipiente de $18 \times 30 \mathrm{~cm}$ ) e aos 140 dias os tratamentos T11 (Substrato orgânico e recipiente de 17x22 cm), T14 (Substrato orgânico e recipiente de $18 \times 30 \mathrm{~cm}$ ) e T17 (Substrato orgânico e recipiente de $25 \times 30 \mathrm{~cm}$ ) e aos 170 dias os tratamentos T14 (Substrato orgânico e recipiente de $18 \times 30 \mathrm{~cm}$ ) e T17 (Substrato orgânico e recipiente de $25 \times 30 \mathrm{~cm}$ ), não houve diferenças significativas entre si, onde foram encontradas as maiores médias de desenvolvimento em diâmetro do colo (tabela 2).

Levando-se em consideração que as maiores médias de diâmetro de colo foram alcançadas nos tratamentos com composto orgânico de esterco bovino + esterco de galinha + capim-mombaça, pode-se inferir que houve uma tendência de que os teores de potássio $(\mathrm{K})$ presentes no substrato contribuíram para 0 crescimento do diâmetro do colo das mudas de Toona ciliata var. australis. 0 potássio $(\mathrm{K})$, além de regular a abertura estomática, promove o engrossamento do caule das mudas, na fase de produção (VALERI \& CORRADINI, 2005).

Segundo LELES et al., (2001) e NOVAES et al., (2002), a altura das mudas e o diâmetro de colo são as características mais importantes para avaliação da sua qualidade, normalmente mudas de maior diâmetro apresentam maior capacidade de emissão de novas raízes obtendo maior sobrevivência inicial no campo. 
TABELA 3: Médias de comprimento radicular (COMR), massa seca da parte aérea da planta (MSA), massa seca radicular (MSR), e Índice Qualidade de Dickson (IQD).

\begin{tabular}{|c|c|c|c|c|}
\hline TRATAMENTO & COMR & MSA (g) & $\operatorname{MSR}(\mathrm{g})$ & IQD \\
\hline $\mathrm{T} 1$ & $0,00 \mathrm{c}$ & $0,00 \mathrm{c}$ & $0,00 \mathrm{c}$ & $0,00 \mathrm{~d}$ \\
\hline T2 & $0,00 \mathrm{c}$ & $0,00 \mathrm{c}$ & $0,00 \mathrm{c}$ & $0,00 \mathrm{~d}$ \\
\hline T3 & $2,50 \mathrm{bc}$ & $0,00 \mathrm{c}$ & $0,00 \mathrm{c}$ & $0,00 \mathrm{~d}$ \\
\hline$\overline{T 4}$ & $0,00 \mathrm{c}$ & $0,00 \mathrm{c}$ & $0,00 \mathrm{c}$ & $0,00 \mathrm{~d}$ \\
\hline T5 & $0,00 \mathrm{c}$ & $0,00 \mathrm{c}$ & $0,00 \mathrm{c}$ & $0,00 \mathrm{~d}$ \\
\hline T6 & $1,50 \mathrm{bc}$ & $0,01 \mathrm{c}$ & $0,01 \mathrm{c}$ & $0,00 \mathrm{~cd}$ \\
\hline T7 & $0,00 \mathrm{c}$ & $0,00 \mathrm{c}$ & $0,00 \mathrm{c}$ & $0,00 \mathrm{~d}$ \\
\hline T8 & $5,70 \mathrm{bc}$ & $0,19 \mathrm{c}$ & $0,08 \mathrm{c}$ & $0,01 \mathrm{~cd}$ \\
\hline T9 & $4,18 \mathrm{bc}$ & $0,01 \mathrm{c}$ & $0,01 \mathrm{c}$ & $0,00 \mathrm{~cd}$ \\
\hline$\overline{T 10}$ & $5,56 \mathrm{bc}$ & $0,02 \mathrm{c}$ & $0,02 \mathrm{c}$ & $0,00 \mathrm{~cd}$ \\
\hline $\mathrm{T} 11$ & $13,55 \mathrm{a}$ & $5,09 \mathrm{~b}$ & $1,49 \mathrm{~b}$ & $0,22 \mathrm{c}$ \\
\hline T12 & $5,20 \mathrm{bc}$ & $0,08 \mathrm{c}$ & $0,03 \mathrm{c}$ & $0,00 \mathrm{~cd}$ \\
\hline$\overline{T 13}$ & $4,16 \mathrm{bc}$ & $0,01 \mathrm{c}$ & $0,01 \mathrm{c}$ & $0,00 \mathrm{~cd}$ \\
\hline $\mathrm{T} 14$ & $17,00 \mathrm{a}$ & $10,14 \mathrm{a}$ & $2,58 \mathrm{a}$ & $0,48 \mathrm{~b}$ \\
\hline T15 & $6,67 \mathrm{~b}$ & $0,03 \mathrm{c}$ & $0,02 \mathrm{c}$ & $0,00 \mathrm{~cd}$ \\
\hline T16 & $4,15 \mathrm{bc}$ & $0,02 \mathrm{c}$ & $0,02 \mathrm{c}$ & $0,00 \mathrm{~cd}$ \\
\hline T17 & $15,21 \mathrm{a}$ & $12,18 \mathrm{a}$ & $3,28 \mathrm{a}$ & $0,70 \mathrm{a}$ \\
\hline T18 & $14,25 \mathrm{a}$ & $0,29 \mathrm{c}$ & $0,12 \mathrm{c}$ & $0,02 \mathrm{~cd}$ \\
\hline Média & 5,95 & 1,99 & 0,54 & 0.11 \\
\hline CV (V\%) & 43,96 & 64,08 & $\overline{85,02}$ & 68,04 \\
\hline
\end{tabular}

As médias seguidas pela mesma letra não diferem estatisticamente entre si pelo teste de Tukey ao nível de $5 \%$ de probabilidade.

As mudas de Toona ciliata var. australis apresentaram massa seca radicular variando entre 0,00 a 3,28 g.planta-1. Observou-se que houve um maior desenvolvimento radicular nos tratamentos T14 (Substrato orgânico e recipiente de $18 \times 30 \mathrm{~cm}$ ) e T17 (Substrato orgânico e recipiente de 25×30 cm), diferenciando-se dos demais (tabela 3 ). Notou-se que estes tratamentos permitiram o crescimento radicular, e o substrato foi capaz de reter água e nutrientes e ainda promover trocas gasosas fundamentais para o crescimento das raízes.

Para o comprimento da raiz, os tratamentos que obtiveram maiores médias foram os T11 (Substrato orgânico e recipiente de $17 \times 22 \mathrm{~cm}$ ), T14 (Substrato orgânico e recipiente de $18 \times 30 \mathrm{~cm}$ ), T17 (Substrato orgânico e recipiente de 25×30 $\mathrm{cm}$ ) e T18 (Substrato comercial e recipiente $25 \times 30 \mathrm{~cm}$ ), onde diferiram significativamente dos demais tratamentos, (tabela 3 ).

Os resultados expressos indicam que provavelmente as características dos tratamentos formados com o composto orgânico esterco bovino + esterco de galinha + capim-mombaça propiciaram maiores ganhos em massa da parte aérea. As mudas que se desenvolveram nos tratamentos T14 (Substrato orgânico e recipiente de $18 \times 30 \mathrm{~cm}$ ) e T17 (Substrato orgânico e recipiente de 25×30 cm) apresentaram maior acúmulo de matéria seca da parte aérea, comparado aos demais tratamentos (tabela 3). 
Deve-se ressaltar que para haver um bom desenvolvimento da raiz é necessário que o substrato apresente boa aeração, facilitando as trocas gasosas e um adequado volume de recipiente. De acordo com BUCKERIDGE et al., (2004), raízes primárias e raízes jovens respiram muito intensamente e para essas raízes, o oxigênio necessário para o processo respiratório, advém do próprio solo (substrato), demonstrando a importância de se observar a porosidade do substrato a ser utilizado.

Diante disto, pode-se inferir que as raízes das mudas de Toona ciliata var. australis obtiveram maior desenvolvimento quando se utilizou recipientes maiores. $O$ resultado foi semelhante ao encontrado por CARNEIRO (1985), trabalhando com Pinus taeda, o qual concluiu que tubetes de maiores dimensões produzem mudas com maiores quantidades de raízes.

Os valores de índice de qualidade de Dickson (IQD) para as mudas de Toona ciliata var. australis variaram em função do volume dos tubetes e sacos plásticos que obtiveram médias entre 0,00 e 0,70 respectivamente (tabela 3), de acordo com os recipientes. Sendo que a maior média de distribuição da biomassa da muda foi obtida no tratamento T17 (Orgânico (25×30)), diferenciando-se estatisticamente dos demais tratamentos.

MALAVASI \& MALAVASI (2006) relataram que ao trabalharem com produção de mudas de duas espécies florestais com a utilização de substrato comercial (GIOPLANT tipo III), que 70 dias após a semeadura, observaram que o tamanho do tubete influenciou nos índices de qualidade de Dickson (IQD), encontrando valores de 0,038 e 0,155 em Cordia trichotomae valores de 0,016 a 0,068, em Jacaranda micranta, em tubetes de 55 e $300 \mathrm{~cm}^{3}$, respectivamente.

Notou-se que o volume do recipiente e a qualidade dos substratos tiveram grande influência no desenvolvimento das mudas de Toona ciliata var. australis, sendo o uso saco de polietileno, em recipiente de maior volume, proporcionou maior desenvolvimento das mudas. Dependendo da qualidade do substrato, os recipientes de maiores volumes normalmente tendem a disponibilizar maiores quantidades de nutrientes e água retida (GOMES et al., 2003).

\section{CONCLUSÃO}

O tratamento que proporcionou os maiores resultados para todas as características analisadas foi o T17, que continha como substrato o composto orgânico (capim-mombaça + esterco de galinha + esterco bovino) e o recipiente saco plástico $(18 \times 30)$.

\section{REFERÊNCIAS}

ABREU, A. H. M.; LELES, P. S. dos. S.; MELO, L. A. de.; FERREIRA, D. H. A. A.; MONTEIRO, F. A. S. Produção de mudas e crescimento inicial em campo de enterolobium contortisiliquum produzidas em diferentes recipientes. Revista Floresta. Curitiba, PR, v. 45, n. 1, p. 141 - 150, jan. / mar. 2015. Disponível em: < http://ojs.c3sl.ufpr.br/ojs/index.php/floresta/article/viewFile/28931/24819>. doi: 10.5380/rf.v45i1.28931.

AJALA, M. C.; AQUINO, N. F.; MALAVASI, U. C.; MALAVASI, M. M. Efeito do volume do recipiente na produção de mudas e no crescimento inicial de Jatropha curcas L. no Oeste Paranaense. Revista Semina Ciências Agrárias. Londrina, v. 
33, n. 6, p. 2039-2046, nov./dez. 2012. Disponível em: <http://dx.doi.org/10.5433/1679-0359.2012v33n6p2039>. doi: 10.5433/1679-0359.

ALMEIDA, N. A.; MENDES, L. M.; OKINO, E. Y. A.; GARLET, A.; MORI, F. A.; MENDES, R. F. Biodeterioração de produtos à base da madeira de Cedro Australiano (Toona ciliata M. Roem. var. australis). Revista Cerne. Lavras, v. 18, n. 1, p. 17-26, jan./mar. 2012. Disponivel em: < http://dx.doi.org/10.1590/S010477602012000100003 >. doi: 10.1590/S0104-77602012000100003.

ALVES, A. S.; OlIVEIRA, L. S. B. de.; ANDRADE, L. A. de.; GONÇAVES, G. S.; SILVA. J. M. da. Produção de mudas de angico em diferentes tamanhos de recipientes e composições de substratos. Revista Verde de Agroecologia e Desenvolvimento Sustentável. Mossoró - RN, v. 7, n. 2, p. 39-44, abr-jun, 2012. Disponível em: <http://www.gvaa.com.br/revista/index.php/RVADS/article/download/1108/1153>.

BARBOSA, T. C.; RODRIGUES, R. B.; COUTO, H. T. Z. Tamanhos de recipientes e o uso de hidrogel no estabelecimento de mudas de espécies florestais nativas. Revista Hoehnea, v. 40, n. 3, p. 537-556, 2013. Disponível em: $<$ http://www.scielo.br/pdf/hoehnea/v40n3/13.pdf >.

BOENE, H. C. A. M.; NOGUEIRA, A. C.; SOUSA, N. J.; KRATZ, D.; SOUZA. P. V. D. de. Efeitos de diferentes substratos na produção de mudas de Sebastiania commersoniana. Floresta, v. 43, n. 3, p. 407-420. 2013. Disponível em: < http://dx.doi.org/10.5380/rf.v43i3.25789 >. doi: 10.5380/rf.v43i3.25789.

BRAZ, R. L.; OLIVEIRA, J. T. da. S.; ARANTES, M. D. C.; RODRIGUES, B. P.; Propriedades físicas e mecânicas da madeira de Toona ciliata em diferentes idades. Floresta, v. 43, n. 4, p. 663-670. 2013. Disponível em: < http://dx.doi.org/10.5380/rf.v43i4.30559>. doi: 10.5380/rf.v43i4.30559.

BUCKERIDGE, M.S.; TINÉ, M.A.S.; MINHOTO, M.J.; LIMA, D.U. Respiração. In: KERBAUY, G.B. (Ed.). Fisiologia vegetal. Rio de Janeiro: Guanabara Koogan, 2004. p.198-216.

CARNEIRO, J. G. A. Efeito da densidade sobre o desenvolvimento de alguns parâmetros morfofisiológicos de mudas de Pinus taeda L. em viveiro e após 0 plantio. Curitiba: UFPR, 140 p. 1985.

COÊLHO, I. A. M.; BOTELHO, A. V. F.; LOPES, I. S.; COÊLHO, O. A. M.; SERPA, P. R. K.; PASSOS, M. A. A. Efeito de recipientes e tipo de substratos na qualidade das mudas de Poincianella pyramidalis (Tul.) L. P. Queiroz. Revista Scientia Plena. v. 9 , n. $5.2013 . \quad$ Disponível em: <http://www.scientiaplena.org.br/sp/article/view/802/707>.

CUNHA, A. O.; ANDRADE, L. A. de.; BRUNO, R. DE. L. A.; SILVA, J. A. L. da.; SOUZA, V. C. de. Efeitos de substratos e das dimensões dos recipientes na qualidade das mudas de Tabebuia impetiginosa (Mart. Ex D.C.) Standl. Revista Arvore. Viçosa-MG, v.29, n.4, p.507-516, 2005. Disponivel em: 
<http://dx.doi.org/10.1590/S0100-67622005000400002>. doi: 10.1590/S010067622005000400002.

DICKSON, A.; LEAF, A.L.; HOSNER, J.F. Quality appraisal of white spruce and white pine seedling stock in nurseries. Forest Chronicle, v. 36, p.10-13, 1960. Disponível em: < http://pubs.cif-ifc.org/doi/abs/10.5558/tfc36010-1>. doi: 10.5558/tfc36010-1.

GOMES, J. M.; COUTO, L.; LEITE, H. G.; XAVIER, A.; GARCIA, S. L. R. Crescimento de mudas de Eucalyptus grandis em diferentes tamanhos de tubetes e fertilização N-P-K. Revista Árvore [online]. v.27, n.2, p.113-127, 2003. Disponível em: <http://dx.doi.org/10.1590/S0100-67622003000200001>. doi: 10.1590/S010067622003000200001.

KLEIN, C. Utilização de substratos alternativos para produção de mudas. Revista Brasileira de Energias Renováveis. v.4, n. 1 p. 43-63, 2015. Disponível em: <http://ojs.c3sl.ufpr.br/ojs/index.php/rber/article/download/40742/pdf_64>.

KRATZ, D.; WENDLLING, I.; NOGUEIRA, A. C.; ZOUZA, P. V. Propriedades físicas e químicas de substratos renováveis. Revista Árvore. Viçosa-MG, v.37, n.6, p.11031113, 2013. Disponível em: <http://dx.doi.org/10.1590/S0100-67622013000600012>. doi: 10.1590/S0100-67622013000600012.

LELES, P. S. S.; CARNEIRO, J. G. de. A.; NOVAES, A. B. de.; BARROSO, D. G. Crescimento e arquitetura radicial de plantas de eucalipto oriundas de mudas produzidas em blocos prensados e em tubetes após o plantio. Revista Cerne. v.7, n.1, p.10-19, 2001.2 Disponível em: $<$ http://www.redalyc.org/articulo.oa?id=74470102>.

LISBOA, A. C.; SANTOS, P. S. dos.; NETO, S. N. de. O.; CASTRO, D. N. de ABREU, A. H. M. de. Efeito do volume de tubetes na produção de mudas de Calophyllum brasiliense e Toona ciliata. Revista Árvore. vol. 36 n. 4 Viçosa Jul/Ago. 2012. Disponível em: <http://dx.doi.org/10.1590/S0100-67622012000400003>. doi: 10.1590/S0100-67622012000400003.

MALAVASI, U. C.; MALAVASI, M. M. Efeito do volume do tubete no crescimento inicial de plântulas de Cordia trichotoma (Vell.) Arrab. Ex Steud e Jacaranda micranta Cham. Revista Ciência Florestal. v.16, n.1, p.11-16, 2006. Disponível em: $<$ http://www.redalyc.org/articulo.oa?id=53416102>.

MIGLIORINI, P. et al. Efeito de diferentes substratos no desenvolvimento inicial de cedro australiano. Revista de Ciências Agroveterinárias. Lages, v.14, n.2, p.139145, $2015 . \quad$ Disponível em: $<$ http://www.revistas.udesc.br/index.php/agroveterinaria/article/view/5858>.

MORETTI, B. S.; NETO, A. E. F.; PINTO, S. I. do. C.; FURTINI, I. V.; MAGALHÃES, C. A. de. S. M. Crescimento e nutrição de mudas de Cedro de Australiano 453 (Toona ciliata) sob omissão de nutrientes. Revista Cerne [online]. v. 17, n. 4, p. 453463, out./dez. 2011. ISSN 0104-7760. Disponível em: 
<http://dx.doi.org/10.1590/S0104-77602011000400003>. doi: 10.1590/S010477602011000400003.

NOVAES, A. B.; CARNEIRO, J. G. de. A.; BARROSO, D. G.; LELES, P. S dos. S. Avaliação do potencial de regeneração de raízes de mudas de Pinus taeda $\mathrm{L}$. produzidas em diferentes tipos de recipientes e o seu desempenho no campo. Revista Árvore [online]. v.26, n.6, p.675-681, 2002. Disponível em: <http://dx.doi.org/10.1590/S0100-67622002000600004>. doi: 10.1590/S010067622002000600004 .

OLIVEIRA, L. S. B. de.; ANDRADE, L. A. de.; ALVES, A. de. S.; GONÇALVES, G. S. Substrato e volume de recipiente na produção de mudas de jatobá (Hymenaea courbaril L.). Revista Nativa, Sinop, v. 02, n. 02, p. 103-107, abr./jun. 2014. Disponível em: <http://dx.doi.org/10.14583/2318-7670.v02n02a07>. doi: 10.14583/2318-7670.

SILVA, A. L.; MORAIS, G. A. Influência de diferentes substratos no crescimento inicial de Ormosia arbórea (Vell.) Harms (Fabaceae). Revista Verde de Agroecologia e Desenvolvimento Sustentável. Mossoró - RN, v. 8, n. 4, p. 22-27, out - dez, 2013. Disponível em: <http://www.gvaa.com.br/revista/index.php/RVADS/article/view/2243/1825>.

SILVA, F. de. A. S., 2015. ASSISTAT: Versão 7.7 beta. DEAG-CTRN-UFCG Atualizado em 01 de abril de 2015. Disponível em: <http://www.assistat.com/>. Acesso em: 10 de jan. de 2016.

SOUZA, V. C.; ANDRADE, L. A. de.; BRUNO, R. de. L. A.; CUNHA, A. de. O. SOUZA, A. P. de. Produção de mudas de ipê-amarelo (Tabebuia serratifolia (Vahl.) Nich.) em diferentes substratos e tamanhos de recipientes. Revista Agropecuária Técnica. v.26, n.2, p.98-108, 2005. Disponível em: <www.cca.ufpb.br/revista/pdf/2005_2_4.pdf>

TUKEY, J. W. Exploratory data analysis. Addison-Wesley Publishing Company, 1977, 688p.

VALERI, S.V.; CORRADINI, L. Fertilização em viveiros para a produção de mudas de Eucalyptus e Pinus. In: GONÇALVES, J.L.M.; BENEDETTI, V. (Ed.). Nutrição e fertilização florestal. Piracicaba: Instituto de Pesquisas e Estudos Florestais. p. 167-190. 2005. 